\title{
Raman-Ramsey CPT with a Grating Magneto-Optical Trap
}

\author{
Rachel Elvin, Gregory W. Hoth, Michael W. Wright, \\ James P. McGilligan, Aidan S. Arnold, Paul F. Griffin and Erling Riis \\ Experimental Quantum Optics and Photonics Group \\ SUPA, Dept. of Physics \\ University of Strathclyde \\ Glasgow, United Kingdom \\ Email: rachel.elvin@strath.ac.uk
}

\begin{abstract}
We describe an experiment which combines cold ${ }^{87} \mathrm{Rb}$ atoms from a grating magneto-optical trap (GMOT) with Lin $\perp$ Lin coherent population trapping (CPT) and pulsed Ramsey interrogation. The bichromatic fields required for $\operatorname{Lin} \perp$ Lin are generated by combining light from a single external cavity diode laser (ECDL) with an electro-optic modulator (EOM) and an acousto-optic modulator (AOM). With this laser system and the GMOT, we are able to produce Raman-Ramsey fringes using either the $F^{\prime}=1$ or the $F^{\prime}=2$ excited states of the ${ }^{87} \mathbf{R b}$ $D_{1}$ line. As a step towards realising a frequency standard based on the GMOT, we measure the Ramsey fringe amplitude as a function of the magnetic bias field and the excited state. We observe dark state interference with $F^{\prime}=1$ and show that this interference is suppressed with $F^{\prime}=2$, as expected from prior work on CPT with ${ }^{87} \mathrm{Rb}$ in thermal vapour cells.
\end{abstract}

\section{INTRODUCTION}

Laser cooling is responsible for many innovations in quantum sensing and atomic metrology [1]. While the pursuit of better performance leads to exciting possibilities [2], it is also important to reduce the complexity of the apparatus in order to bring the benefits of cold atoms to more applications. The majority of compact atomic clocks are based on room temperature systems; however, the long-term stability is limited by collisional shifts from buffer gases. This can be overcome through the use of cold atoms, which provide long interaction times for better sensitivity. The grating magneto-optical trap (GMOT) is a favourable candidate for a compact cold-atom clock, due to its relative simplicity and potential for further miniaturisation [3]-[5].

Optical interrogation of the hyperfine ground state splitting can be done using the coherent population trapping (CPT) technique [6], [7]. In CPT, a bichromatic laser field is used to pump atoms into a non-interacting superposition of the clock states via one excited state. By monitoring the transmission of the laser field through an atomic sample, one can use these 'dark' resonances as narrow linewidth frequency references without the need for $\mathrm{cm}$-scale microwave cavities.

The frequency resolution of the CPT resonances can be further improved through the use of a two pulse RamanRamsey sequence [8]. In the first pulse, the atoms are pumped into a dark state. Then, the atoms are left to freely precess for a time $T$. During this free evolution time, the phase builds up between the driving CPT field and the atomic coherence. A second pulse of the CPT light maps the accumulated phase into absorption, and interference fringes with a width of $1 / T$ are observed inside a Lorentzian envelope when the transmission of the readout pulse is measured.

For a high-performance frequency reference, it is desirable to maximise the contrast of the CPT resonance while minimising its sensitivity to external influences. Through extensive studies, several techniques have been developed to satisfy this requirement, including $\operatorname{Lin} \| \operatorname{Lin}[9]-[11]$, Lin $\perp$ Lin [8], [12], and Push-Pull Optical Pumping [13]-[15]. With these methods, high-contrast CPT resonances are realised by arranging for the constructive interference of multiple dark resonances. However, this can also introduce unwanted magnetic sensitivities. With Raman-Ramsey interrogation, this can manifest as oscillations in the fringe amplitude as a function of the magnetic field used to define the quantisation axis [10], [16], [17]. In the case of the $D_{1}$ line of ${ }^{87} \mathrm{Rb}$, these interference effects depend strongly on the excited state chosen to drive the CPT resonance [18]. With $F^{\prime}=1$, three dark resonances are expected to be present leading to large interference in the Ramsey fringe amplitude. With $F^{\prime}=2$, two of these dark resonances are suppressed by couplings to the $\left|F^{\prime}=2, m_{F}= \pm 2\right\rangle$ states and so the magnetic sensitivity of the Ramsey fringe amplitude will also be reduced [18]. These dark state interference effects can degrade the performance of the CPT resonance as a frequency standard, and so it is important to characterise them experimentally [16], [17].

Here, we describe our apparatus and an initial characterisation of these dark state interference effects in our system. In section II, we describe the laser system used for Lin $\perp$ Lin CPT. In section III, we review the three dark resonances that arise in $\operatorname{Lin} \perp$ Lin CPT with $F^{\prime}=1$. In section IV, we describe the Ramsey fringes produced in our apparatus with an interrogation time $T=9 \mathrm{~ms}$ as a function of the excited state and the bias field. The paper concludes with section V.

\section{LASER SYSTEM FOR LIN $\perp$ LIN CPT}

This section describes the laser system in the clock side of the experiment and details the preparation of a laser spectrum 


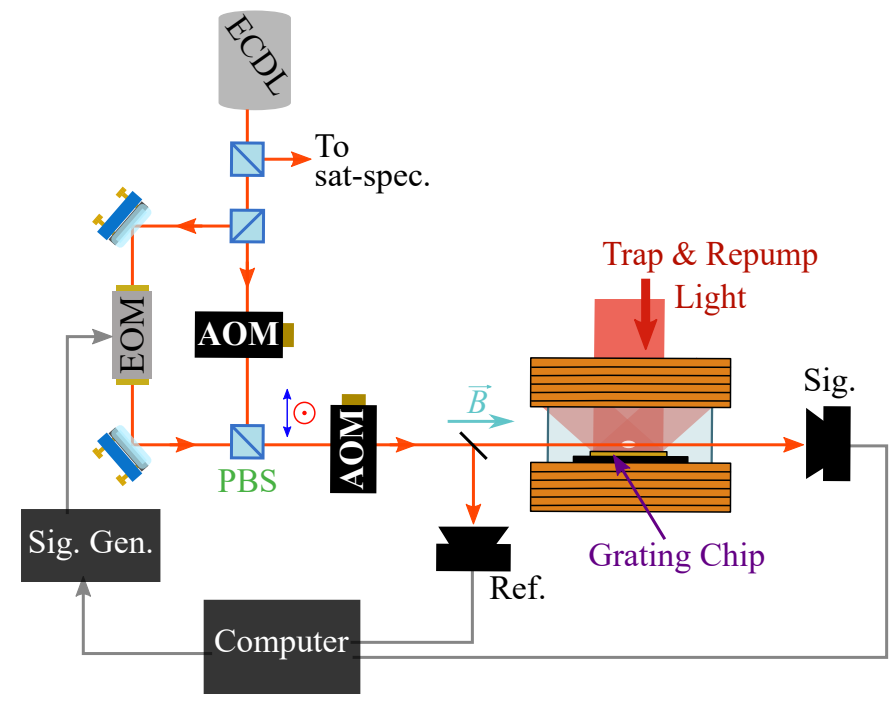

Fig. 1. Laser system and simplified optical layout for the clock side of the experiment. ECDL: external cavity diode laser. EOM: electro-optical modulator. AOM: acousto-optical modulator. PBS: polarising beam splitter. Magnetic bias field direction is denoted by $\vec{B}$. Two photodetectors, 'Ref.' and 'Sig.', measure the laser intensity before and after the atoms respectively.

appropriate for Lin $\perp$ Lin CPT with light from one external cavity diode laser (ECDL).

In the cooling and trapping side of the experiment, homebuilt ECDLs are used to generate trap and re-pump light on the ${ }^{87} \mathrm{Rb} \mathrm{D}_{2}(780 \mathrm{~nm})$ line. The combined light is then fibrecoupled onto the grating chip which is held outside the glass cell of the vacuum chamber. With the GMOT, we are able to load more than $10^{7}$ atoms with one beam coupled to a $2 \times 2 \mathrm{~cm}$ grating chip [19]. The GMOT has also recently realised atom temperatures as low as $3 \mu \mathrm{K}$ [20].

A simplified diagram of our CPT experiment is shown in Fig. 1. We use a $795 \mathrm{~nm}$ ECDL and a fibre-coupled electrooptical phase modulator (EOM) to address the $D_{1}$ line of ${ }^{87} \mathrm{Rb}$. The orthogonal linearly polarised components for Lin $\perp$ Lin CPT originate from one laser, and are therefore inherently phase-coherent. The $\mathrm{D}_{1}$ ECDL is locked using saturated absorption spectroscopy, and the frequency of the locked laser is made tunable by use of an AOM in the spectroscopy path (not shown in Fig. 1).

The primary beam path is split into two arms using a polarising beam splitter (PBS). The light through one of the arms is passed through the EOM which is driven by a microwave signal with a frequency of $f_{0}+\delta$, where $f_{0} \approx 6.834 \mathrm{GHz}$ is tunable about the ground-state hyperfine splitting of ${ }^{87} \mathrm{Rb}$ and $\delta \approx 200 \mathrm{MHz}$ is a fixed frequency offset. In the second arm the light is passed through an acousto-optical modulator (AOM) operating at $f=\delta$. The beams are then recombined on a PBS. At this point, the light that passed through the AOM and a single sideband of the light that passed through the EOM are now in two-photon resonance with the atoms, in addition to being orthogonally polarised. A second AOM is used for temporal control of pulses and to bring light from both arms onto the single photon resonance. With the appropriate choice

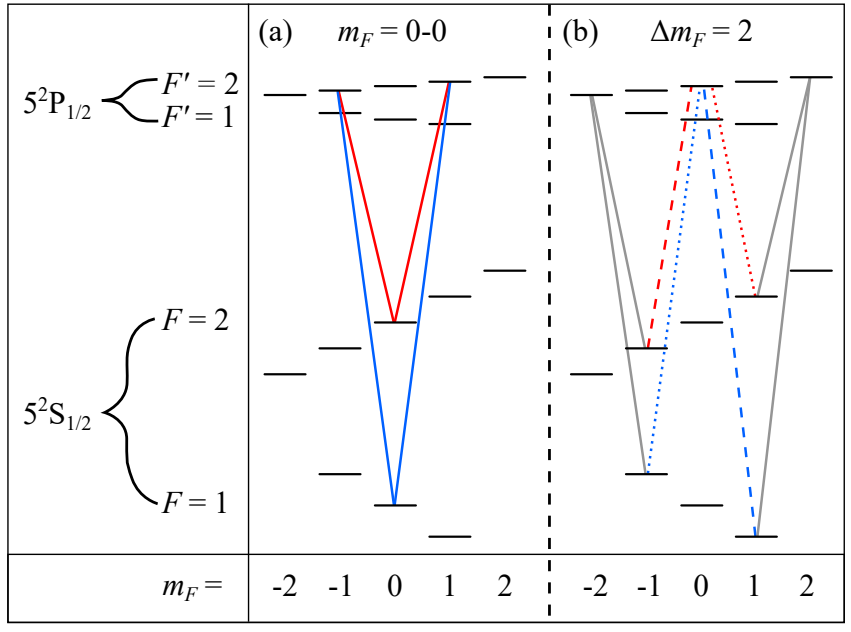

Fig. 2. CPT resonances present in the Lin $\perp$ Lin scheme for the ${ }^{87} R b D_{1}$ line. The solid red and blue lines in (a) show the transitions that pump atoms into the coherent dark state with $m_{F}=0-0$. The red and blue lines in (b) show the (-1) - (+1) and (+1) - (-1) transitions, as dashed and dotted lines respectively. These latter transitions pump atoms into coherent dark states referred to as $\Delta m_{F}=2$. The outer grey lines in (b) highlight the transitions which suppress the $\Delta m_{F}=2$ dark states.

of AOM offset frequencies and lock-points, we are able to operate the system with the CPT pair resonant with either $F^{\prime}=1$ or $F^{\prime}=2$.

The use of the EOM results in additional off-resonant light, with components that are detuned by $\delta$ and by $f_{\text {hfs }}+\delta$ from the clock resonance. We plan to investigate the light shifts caused by these off-resonant components in future work.

A magnetic bias of $\sim 100 \mathrm{mG}$ along the direction of the laser propagation, generated by a Helmholtz coil pair, is used to set the quantisation axis and to lift the degeneracy on the magnetic (Zeeman) sub-levels. The transmission of the laser light is measured by two photodetectors, one before and one after the atoms. A computer records both signals from the probe pulse, which enables normalisation of the signal to minimise the effect of intensity fluctuations.

\section{DARK State Structure IN Lin $\perp$ LiN}

In this section, we review the dark resonances that play an important role in a Lin $\perp$ Lin CPT frequency reference [16], [17]. First, a dark resonance can be formed with the $m_{F}=0$ ground states. These states are simultaneously addressed by both circular components of the bi-chromatic CPT beam as shown in Fig. 2 (a). In the Lin $\perp$ Lin configuration, the phases of the polarisation components and the Clebsch-Gordan coefficients are such that the coherent dark state formed by the $\sigma^{+}$pair and the $\sigma^{-}$pair are the same. As a result, a high contrast dark resonance is formed [7], [8]. Since this dark resonance involves only the $m_{F}=0$ ground states, it experience a quadratic Zeeman shift. For ${ }^{87} \mathrm{Rb}$, the shift is given by $\Delta f=575 \mathrm{~Hz} / \mathrm{G}^{2}[21]$.

Two other dark resonances can be formed between the states $\left|F=1, m_{F}= \pm 1\right\rangle$ and $\left|F=2, m_{F}=\mp 1\right\rangle$, as shown in Fig. 2 (b). These $\Delta m_{F}=2$ resonances have a small linear 

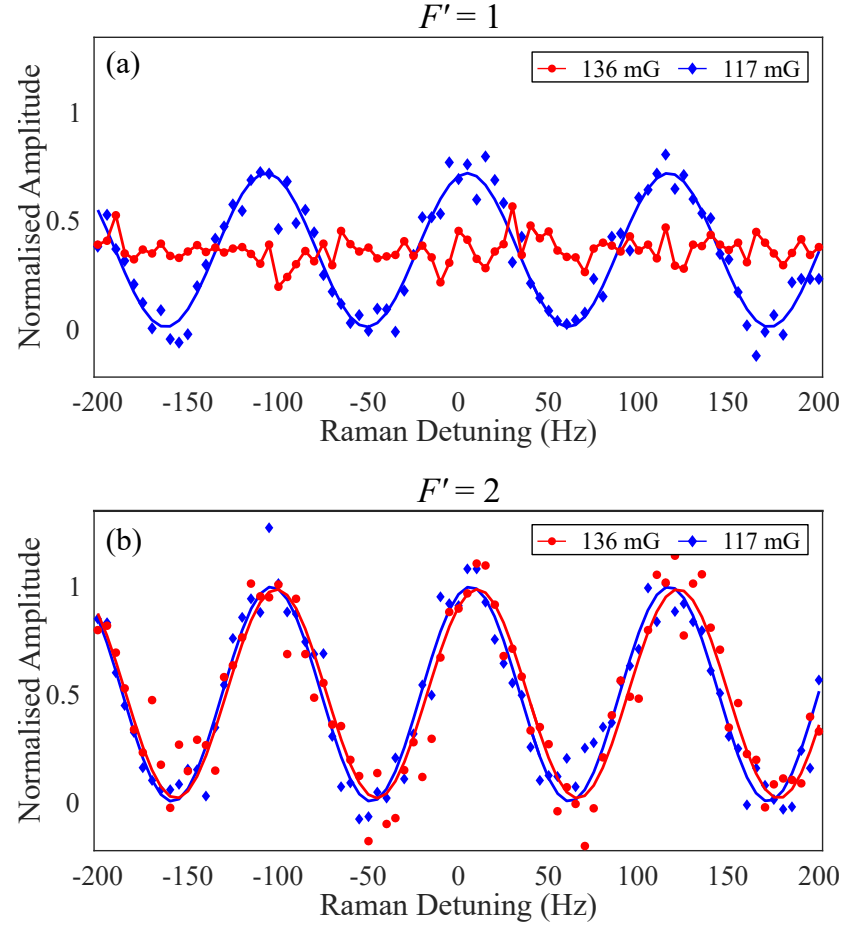

Fig. 3. Ramsey fringes for $\operatorname{Lin} \perp$ Lin $C P T$ when driven via $F^{\prime}=1$ (a) and $F^{\prime}=2$ (b), for magnetic bias fields of $B=136 \mathrm{mG}$ and $B=117 \mathrm{mG}$. In both cases, the first CPT pulse was $200 \mu$ s long and the free evolution time was $T=9 \mathrm{~ms}$. The fringe amplitude was normalised to the average amplitude observed with $F^{\prime}=2$.

Zeeman shift due to the nuclear part of the Landé g-factor [9]. For ${ }^{87} \mathrm{Rb}$, the shift of the $\Delta m_{F}=2$ resonances is given by $\Delta f=432 \mathrm{~Hz} / \mathrm{G}^{2} \pm 2786 \mathrm{~Hz} / \mathrm{G}[10]$.

The $\Delta m_{F}=2$ resonances are truly dark states only when $F^{\prime}=1$ [9]. When $F^{\prime}=2$, they are suppressed by the coupling to the $\left|F^{\prime}=2, m_{F}= \pm 2\right\rangle$ states as illustrated by the grey lines in Fig. 2 (b) [18]. However, it is possible to produce a coherence associated with the $\Delta m_{F}=2$ dark resonances even when $F^{\prime}>1$ [16]-[18]. Therefore, it is important to characterise the dark state population experimentally.

\section{EXPERIMENT: DARK STATE INTERFERENCE}

In order to explore the dark state interference effects in our apparatus, we measured CPT Ramsey fringes as a function of the magnetic field for both $F^{\prime}=1$ and $F^{\prime}=2$. Ramsey fringes with $T=9 \mathrm{~ms}$ and two different magnetic fields are shown in Fig. 3. With $F^{\prime}=1$, shown in Fig. 3 (a), the fringe pattern washes out when the field changes by approximately $20 \mathrm{mG}$. In the case of $F^{\prime}=2$, this change in the bias field has almost no effect on the fringes.

The variation of fringe amplitude is shown over a wider range of magnetic fields in Fig. 4. The data are consistent with our expectations in that we see high contrast interference with $F^{\prime}=1$, and this interference is suppressed with $F^{\prime}=2$. With the three dark states described in section III, one expects a full cycle of the interference pattern to correspond to a magnetic field change $\Delta B=1 / T Z$ where $Z$ is the residual linear

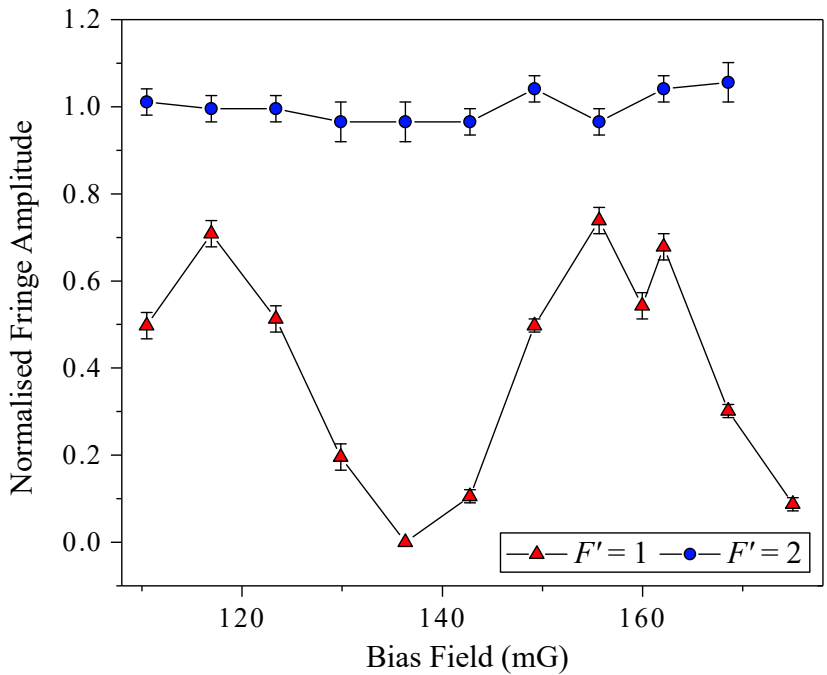

Fig. 4. Ramsey fringe amplitude as a function of the magnetic bias field for $F^{\prime}=1$ (red triangles) and $F^{\prime}=2$ (blue circles). The bias field was calibrated by spectroscopy of the magnetically sensitive CPT resonances. The free evolution time is $T=9 \mathrm{~ms}$. The fringe amplitude was normalised to the average amplitude observed with $F^{\prime}=2$.

Zeeman shift of the $\Delta m_{F}=2$ resonances. For ${ }^{87} \mathrm{Rb}$ and $T=9 \mathrm{~ms}, \Delta B \approx 40 \mathrm{mG}$, which is in good agreement with the data shown in Fig. 4. The nearly complete extinction of the fringes with $F^{\prime}=1$ indicates that roughly half of the population is in the $0-0$ dark state with the other half divided between the two $\Delta m_{F}=2$ dark states, but this simple analysis does not account for the different quadratic Zeeman shifts of the $0-0$ and the $\Delta m_{F}=2$ resonances or an imbalance in the population of the $\Delta m_{F}=2$ dark states.

In the case of $F^{\prime}=2$, one can see that the maximum fringe amplitude is larger by a factor of 1.3 in Fig. 4. A larger contrast enhancement for $F^{\prime}=2$ compared to $F^{\prime}=1$ has been observed for $\operatorname{Lin} \perp$ Lin CPT on the $\mathrm{D}_{1}$ line of ${ }^{87} \mathrm{Rb}$ using continuous interrogation in a thermal vapour [18]. The strong suppression of the interference with $F^{\prime}=2$ is also of interest in its own right. Simulations of Lin $\perp$ Lin CPT in a thermal vapour of ${ }^{87} \mathrm{Rb}$ show that residual excitation of the $\Delta m_{F}=2$ resonances is expected for $F^{\prime}=2$ [18], and it is possible that this could be detected in our apparatus with an improved signal to noise ratio.

\section{CONClusion}

We have shown that GMOT technology can be used as a compact source of cold atoms for CPT interrogation. We have also made an initial study of the magnetic field dependence of the Ramsey fringes in our apparatus with $F^{\prime}=1$ and $F^{\prime}=2$. As expected, with $F^{\prime}=1$, we see significant interference between the three dark states. With $F^{\prime}=2$, the Ramsey fringes are significantly less sensitive to the bias field and the fringe contrast is also increased. Thus, the $F^{\prime}=2$ excited state is a more favorable candidate for realising a frequency reference based on Lin $\perp$ Lin CPT and cold ${ }^{87} \mathrm{Rb}$ atoms. In future work, we plan to investigate the distribution of the dark 
state populations in more detail. Cold ${ }^{87} \mathrm{Rb}$ is a promising system for this study due to its relative simplicity and the availability of an excited state with $F^{\prime}=1$. In the long term, our goal is to demonstrate a frequency reference with good long-term stability based on the GMOT technology.

\section{ACKNOWLEDGMENT}

We acknowledge financial support from EPSRC (EP/M013294/1), DSTL (DSTLX-100095636R), the UK Quantum Technology Hub for Sensors and Metrology and the National Physical Laboratory. We would also like to thank Elizabeth Donley for helpful technical discussions. The research data supporting this publication can be accessed at: $\quad$ http://dx.doi.org/10.15129/64d52d65-b1d5-4466-ad6a$11 \mathrm{c} 2 \mathrm{a} 2 \mathrm{f} 7 \mathrm{c} 6 \mathrm{e} 9$.

\section{REFERENCES}

[1] G. K. Campbell and W. D. Phillips, "Ultracold atoms and precise time standards," Phil. Trans. R. Soc. A, vol. 369, no. 1953, p. 4078, 2011.

[2] A. D. Ludlow, M. M. Boyd, J. Ye, E. Peik, and P. Schmidt, "Optical atomic clocks," Rev. of Mod. Phys., vol. 87, no. 2, pp. 637-701, 2015.

[3] J. P. McGilligan, P. F. Griffin, E. Riis, and A. S. Arnold, "Phase-space properties of magneto-optical traps utilising micro-fabricated gratings," Opt. Express, vol. 23, no. 7, p. 8948, 2015.

[4] J. P. McGilligan, P. F. Griffin., E. Riis, and A. S. Arnold, "Diffractiongrating characterization for cold-atom experiments," J. Opt. Soc. Am. B, vol. 33 , no. 6 , p. 1271, 2016.

[5] J. P. McGilligan, R. Elvin, P. F. Griffin, E. Riis, and A. S. Arnold, "Utilising diffractive optics towards a compact, cold atom clock," in 2016 European Frequency and Time Forum (EFTF), 2016, pp. 1-2.

[6] J. Vanier, "Atomic clocks based on coherent population trapping: a review," Appl. Phys. B, vol. 81, no. 4, pp. 421-442, Aug 2005.

[7] V. Shah and J. Kitching, "Chapter 2 - advances in coherent population trapping for atomic clocks," in Advances in Atomic, Molecular, and Optical Physics. Academic Press, 2010, vol. 59, pp. 21 - 74.

[8] T. Zanon, S. Guerandel, E. de Clercq, D. Holleville, N. Dimarcq, and A. Clairon, "High contrast Ramsey fringes with coherent-populationtrapping pulses in a double lambda atomic system," Phys. Rev. Lett., vol. 94, no. 19, p. 193002, 2005.

[9] A. V. Taichenachev, V. I. Yudin, V. L. Velichansky, and S. A. Zibrov, "On the unique possibility of significantly increasing the contrast of dark resonances on the $\mathrm{D}_{1}$ line of ${ }^{87} \mathrm{Rb}$," J. Exp. Theor. Phys., vol. 82, no. 7, pp. 398-403, 2005.

[10] F.-X. Esnault, E. Blanshan, E. N. Ivanov, R. E. Scholten, J. Kitching, and E. A. Donley, "Cold-atom double- $\Lambda$ coherent population trapping clock," Phys. Rev. A, vol. 88, no. 4, p. 042120, 2013.

[11] X. Liu, E. Ivanov, V. I. Yudin, J. Kitching, and E. A. Donley, "Lowdrift coherent population trapping clock based on laser-cooled atoms and high-coherence excitation fields," Phys. Rev. Appl., vol. 8, no. 5, p. 054001, 2017.

[12] C. Xi, Y. Guo-Qing, W. Jin, and Z. Ming-Sheng, "Coherent population trapping-ramsey interference in cold atoms," Chinese Phys. Lett., vol. 27, no. 11, p. 113201, 2010.

[13] Y.-Y. Jau, E. Miron, A. B. Post, N. N. Kuzma, and W. Happer, "Push-pull optical pumping of pure superposition states," Phys. Rev. Lett., vol. 93, no. 16, p. 160802, 2004.

[14] M. Abdel Hafiz and R. Boudot, "A coherent population trapping Cs vapor cell atomic clock based on push-pull optical pumping," J. Appl. Phys., vol. 118, no. 12, p. 124903, 2015.

[15] M. Abdel Hafiz, G. Coget, P. Yun, S. Guerandel, E. de Clercq, and R. Boudot, "A high-performance raman-ramsey Cs vapor cell atomic clock," J. Appl. Phys., vol. 121, no. 10, p. 104903, 2017.

[16] R. Boudot, S. Guerandel, E. de Clercq, N. Dimarcq, and A. Clairon, "Current status of a pulsed CPT Cs cell clock," IEEE Trans. Instr. Meas., vol. 58, no. 4, pp. 1217-1222, April 2009.

[17] O. Kozlova, J. M. Danet, S. Gurandel, and E. de Clercq, "Limitations of long-term stability in a coherent population trapping Cs clock," IEEE Trans. Instr. Meas., vol. 63, no. 7, pp. 1863-1870, July 2014.
[18] Z. Warren, M. S. Shahriar, R. Tripathi, and G. S. Pati, "Experimental and theoretical comparison of different optical excitation schemes for a compact coherent population trapping Rb vapor clock," Metrologia, vol. 54, no. 4, pp. 418-431, 2017.

[19] C. C. Nshii, M. Vangeleyn, J. P. Cotter, P. F. Griffin, E. A. Hinds, C. N. Ironside, P. See, A. G. Sinclair, E. Riis, and A. S. Arnold, "A surface-patterned chip as a strong source of ultracold atoms for quantum technologies," Nat. Nanotechnol., vol. 8, no. 5, pp. 321-324, 2013.

[20] J. P. McGilligan, P. F. Griffin, R. Elvin, S. J. Ingleby, E. Riis, and A. S. Arnold, "Grating chips for quantum technologies," Sci. Reports, vol. 7, no. $1,2017$.

[21] D. A. Steck, "Alkali D line data," 2015. [Online]. Available: http://steck.us/alkalidata 